\title{
BCS AND ATTRACTIVE HUBBARD MODEL COMPARATIVE STUDY
}

\author{
Nathan Salwen, Steven A. Sheets and Stephen R. Cotanch \\ Department of Physics, North Carolina State University, Raleigh, NC 27695-8202
}

(Dated: November 20, 2018)

\begin{abstract}
We extend previous studies of the BCS canonical approach for the attractive Hubbard model. A derivation of the BCS formulation is presented for both the Hubbard and a simpler reduced Hamiltonian. Using direct diagonalization, exact one and two dimensional solutions for both Hamiltonians are compared to BCS variational calculations. Approximate and exact ground state energies and energy gaps are computed for different electron number systems as well as correlation observables not previously predicted. Reproducing published one dimensional findings, the BCS is an excellent approximation for the Hubbard ground state energy but not energy gap, a finding that remains true in two dimensions. Propagators and correlators are found more sensitive to wavefunctions and appreciable differences are computed with the Hubbard model exhibiting a weaker degree of superconductivity than the BCS. However for the reduced Hamiltonian model the BCS is an excellent approximation for all observables in both one and two dimensions.

PACS numbers: PACS number(s): 74.20.Fg, 71.24.+q, 71.10Fd, 71.10Li
\end{abstract}

\section{INTRODUCTION}

Since the advent of the theory of superconductivity, developed by Bardeen, Cooper and Schrieffer $[\mathrm{BCS}]^{1}$ and their contemporaries, Bogoliubov ${ }^{2}$ and Valatin ${ }^{3}$, countless investigations with these formalisms have been performed in a variety of fields. This widespread application of the BCS approach is due not only to its power and utility but also because superior approximate or exact solutions are generally not available. This is especially true in nuclear ${ }^{4}$ and hadronic structure ${ }^{-5}$ where physical insight has been limited by uncertainties due to approximations. Hence, further assessing the validity of the BCS approximation is an important endeavor with broad attending interest. In particular, there have been several studies 6.7 .8 .9 of the BCS approximation within the Hubbard model for which exact solutions, such as those provided by the Bethe Ansatz ${ }^{10}$, are known but only in one dimension. The purpose of this paper is to extend these investigations in several new directions: 1) assess the BCS approximation for not only the Hubbard but also a reduced Hubbard-like Hamiltonian; 2) in addition to energies and energy gaps also examine propagators and correlation observables; 3 ) evaluate the BCS accuracy in two dimensions.

In discussing the Hubbard model it is natural to restrict ourselves to a fixed filling because the Hamiltonian conserves electron number. Therefore we utilize the canonical BCS formulation with a fixed number of electrons. This is superior to the grand canonical approach which only constrains the average electron number and also produces a higher ground state energy. A previous pioneering study ${ }^{6}$ notably detailed the excellent ground state energy agreement between the exact Hubbard model and the approximate canonical BCS solution. However that work also determined, depending upon electron density, that the Hubbard and BCS energy gaps do deviate, again in one dimension. Related, an odd filling BCS application ${ }^{8}$ in one dimension also found somewhat less precise results. Because the BCS approximation is conjectured to improve in higher dimension ${ }^{6}$, it is important to perform a numerical assessment in two dimensions.

When computing with the BCS ground state many of the terms in the Hubbard Hamiltonian, $H$, become irrelevant since only components which are either diagonal in momentum space or create and destroy momentum modes in pairs contribute. Truncation to these remnant terms defines the reduced Hamiltonian, $H_{r}$, which is specified in the next section where the BCS Hubbard ground state energy, $E_{B C S}$, is found degenerate with the BCS reduced Hamiltonian value. Because $H$ contains further terms describing additional dynamics we a priori expect $E_{B C S}$ to better approximate the exact reduced Hamiltonian ground state energy, $E_{r}$, than the exact Hubbard ground state energy, $E$. Indeed, as we detail below $E_{B C S}$ is an order of magnitude closer to $E_{r}$ than $E$ even though the variational treatment is very good for both. Consequently a more complete BCS assessment, especially for systems modeled by $H_{r}$, should address other observables which, in contrast to energies, are more sensitive to wavefunction details. Accordingly, we also calculate electron pair propagators, wavefunction inner products and $s, p$ and $d$-wave correlators which are important for establishing superconductivity.

This paper is divided into four sections. In section II the Hubbard and reduced Hamiltonians are introduced and a new formulation of the canonical BCS method is derived. This section also details formulas for energy gaps, densities, propagators and $s, p$ and $d$-wave correlators. Section III compares and discusses numerical results for a variety of electron level fillings and different number of lattice sites. Finally, conclusions are summarized in section IV. 


\section{THEORETICAL FORMULATION}

\section{A. Model Hamiltonians}

We follow the literature notation, especially Refs. 6 and 8, and recall the full Hubbard Hamiltonian in momentum space

$$
H=\sum_{\mathbf{p} \sigma} \epsilon_{\mathbf{p}} a_{\mathbf{p} \sigma}^{\dagger} a_{\mathbf{p} \sigma}+\frac{U}{V} \sum_{\mathbf{p p}^{\prime} \mathbf{q}} a_{\mathbf{p} \uparrow}^{\dagger} a_{-\mathbf{p}+\mathbf{q} \downarrow}^{\dagger} a_{-\mathbf{p}^{\prime}+\mathbf{q} \downarrow} a_{\mathbf{p}^{\prime} \uparrow} .
$$

The reduced Hamiltonian retains the non-diagonal $(\mathbf{p} \neq$ $\left.\mathbf{p}^{\prime}\right)$ terms with $\mathbf{q}=0$ and diagonal $\left(\mathbf{p}=\mathbf{p}^{\prime}\right)$ terms

$$
\begin{aligned}
H_{r}=\sum_{\mathbf{p} \sigma} \epsilon_{\mathbf{p}} a_{\mathbf{p} \sigma}^{\dagger} a_{\mathbf{p} \sigma} & +\frac{U}{V} \sum_{\mathbf{p}^{\prime}, \mathbf{p} \neq \mathbf{p}^{\prime}} a_{\mathbf{p} \uparrow}^{\dagger} a_{-\mathbf{p} \downarrow}^{\dagger} a_{-\mathbf{p}^{\prime} \downarrow} a_{\mathbf{p}^{\prime} \uparrow} \\
& +\frac{U}{V} \sum_{\mathbf{p q}} a_{\mathbf{p} \uparrow}^{\dagger} a_{-\mathbf{p}+\mathbf{q} \downarrow}^{\dagger} a_{-\mathbf{p}+\mathbf{q} \downarrow} a_{\mathbf{p} \uparrow}
\end{aligned}
$$

In the above equations $a_{\mathbf{p} \sigma}^{\dagger}\left(a_{\mathbf{p} \sigma}\right)$ creates (annihilates) an electron with momentum $\mathbf{p}$ and spin $\sigma=\uparrow$ or $\downarrow$. In one dimension the electron kinetic energy is $\epsilon_{\mathbf{p}}=-2 t \cos (\mathbf{p})$, while in two dimensions, $\epsilon_{\mathbf{p}}=-2 t\left(\cos \left(p_{x}\right)+\cos \left(p_{y}\right)\right)$, with hopping rate $t$ (set to 1 in this work) between nearest neighbors. The momenta are restricted to multiples of $\frac{2 \pi}{L}$, where $L$ is the linear extent of the system. The number of sites $V$ is $L$ in one dimension or $L^{2}$ in two dimensions. Note that the last term in Eq. (2) can be expressed in terms of a sum over the dummy index $\mathbf{l}=-\mathbf{p}+\mathbf{q}$ yielding $\frac{U}{V} N_{e \uparrow} N_{e \downarrow}$, the product of spin up, $N_{e \uparrow}$, and down, $N_{e \downarrow}$, electron number operators for a system having $V$ total lattice sites. Since we restrict this discussion to the attractive Hubbard model the potential coupling parameter $U$ is negative. The reduced Hamiltonian, $H_{r}$, has been chosen to only contain elements of $H$ which have non-zero expectation values in the BCS state defined in the next section.

\section{B. BCS Approximation}

We adopt the formulation of Ref. 6 but present an alternate BCS derivation. For a given Hamiltonian, the BCS approximation entails using the unnormalized variational wavefunction

$$
|B C S\rangle=\prod_{\mathbf{k}}\left(1+g_{\mathbf{k}} a_{\mathbf{k} \uparrow}^{\dagger} a_{-\mathbf{k} \downarrow}^{\dagger}\right)|0\rangle,
$$

to minimize the ground state energy. Here $g_{\mathbf{k}}$ are the variational parameters and $|0\rangle$ represents the vacuum state with no electrons. Note that the above Hamiltonians conserve particle number, but the state $|B C S\rangle$ includes a mixture of fock components with different particle number. Because we are interested in states with fixed fillings we organize these components by electron number $N_{e}=2 \nu$ with $\nu=0,1,2,3 \ldots$

$$
|B C S\rangle=\sum_{\nu}\left|\Psi_{2 \nu}\right\rangle
$$

$$
\left|\Psi_{2 \nu}\right\rangle=\sum_{s(\nu:)} \prod_{i=1}^{\nu} g_{\mathbf{k}_{i}} a_{\mathbf{k}_{i} \uparrow}^{\dagger} a_{-\mathbf{k}_{i \downarrow} \downarrow}^{\dagger}|0\rangle .
$$

Here and below the cryptic summation notation $s(\nu:)$ is used to represent the sum over all unordered sets of momentum coordinates, $\mathbf{k}_{1}, \mathbf{k}_{2}, \ldots \mathbf{k}_{\nu}$, with the restriction that all of the momenta are different.

For a physical system with $N_{e}$ electrons the BCS ground state energy, $E_{B C S}$, is calculated by minimizing the expectation value $E_{2 \nu}$

$$
E_{2 \nu} \equiv \frac{\left\langle\Psi_{2 \nu}|H| \Psi_{2 \nu}\right\rangle}{\left\langle\Psi_{2 \nu} \mid \Psi_{2 \nu}\right\rangle}
$$

i.e. $E_{B C S}=\min E_{2 \nu}$ where the minimization is achieved by varying the $g_{\mathbf{k}}$ as detailed below.

Before proceeding several points are in order. First, in the grand canonical BCS formulation the ground state energy is obtained by minimizing $\frac{\langle B C S|H| B C S\rangle}{\langle B C S \mid B C S\rangle}$. Using Eq. (3) one can show that the grand canonical is greater than the minimum canonical energy. Second, combining Eqs. (125) directly yields the degenerate result

$$
\frac{\left\langle\Psi_{2 \nu}|H| \Psi_{2 \nu}\right\rangle}{\left\langle\Psi_{2 \nu} \mid \Psi_{2 \nu}\right\rangle}=\frac{\left\langle\Psi_{2 \nu}\left|H_{r}\right| \Psi_{2 \nu}\right\rangle}{\left\langle\Psi_{2 \nu} \mid \Psi_{2 \nu}\right\rangle}
$$

verifying, as stated above, that the BCS ground state energy is the same for both Hamiltonians. This is also true for the grand canonical BCS energy. Third, the successive operation of $H_{r}$ on $|0\rangle$ only generates states having electrons paired just like the state $|\mathrm{BCS}\rangle$ with opposite momentum and spin, called Cooper pairs. In general, the exact Hubbard ground state will contain additional electron correlations between such pairs and the BCS approach assumes these correlations can be ignored.

Now, we compute the norm of the fock state

$$
\left\langle\Psi_{2 \nu} \mid \Psi_{2 \nu}\right\rangle=\sum_{s(\nu:)} \prod_{i=1}^{\nu} g_{\mathbf{k}_{i}}^{2}
$$

and the unnormalized kinetic energy

$$
\left\langle\Psi_{2 \nu}\left|\sum_{p \sigma} \epsilon_{\mathbf{p}} a_{\mathbf{p} \sigma}^{\dagger} a_{\mathbf{p} \sigma}\right| \Psi_{2 \nu}\right\rangle=2 \sum_{\mathbf{p}} \epsilon_{\mathbf{p}} g_{\mathbf{p}}^{2} \sum_{s(\nu-1: \mathbf{p})} \prod_{i}^{\nu-1} g_{\mathbf{k}_{i}}^{2} .
$$

The modified summation index $s\left(n: \mathbf{p}_{1}, \mathbf{p}_{2} \ldots\right)$ now denotes the sum over all unordered sets of momentum coordinates, $\mathbf{k}_{1}, \mathbf{k}_{2}, \ldots \mathbf{k}_{n}$, with the stronger restriction that all momenta in the extended set, $\left\{\mathbf{k}_{1}, \ldots \mathbf{k}_{n}, \mathbf{p}_{1}, \mathbf{p}_{2}, \ldots\right\}$, are different. For the potential energy we first calculate

$$
\begin{aligned}
& \sum_{\mathbf{p}} a_{-\mathbf{p} \downarrow} a_{\mathbf{p} \uparrow}\left|\Psi_{2 \nu}\right\rangle \\
& =\sum_{\mathbf{p}} a_{-\mathbf{p} \downarrow} a_{\mathbf{p} \uparrow} \sum_{s(\nu:)} \prod_{i=1}^{\nu} g_{\mathbf{k}_{i}} a_{\mathbf{k}_{i} \uparrow}^{\dagger} a_{-\mathbf{k}_{i} \downarrow}^{\dagger}|0\rangle \\
& =\sum_{\mathbf{p}} g_{\mathbf{p}} \sum_{s(\nu-1: \mathbf{p})} \prod_{i=1}^{\nu-1} g_{\mathbf{k}_{i}} a_{\mathbf{k}_{i} \uparrow}^{\dagger} a_{-\mathbf{k}_{i \downarrow}}^{\dagger}|0\rangle .
\end{aligned}
$$


Then the unnormalized potential energy matrix element is

$$
\begin{gathered}
\left\langle\Psi_{2 \nu}\left|\frac{U}{V}\left(\sum_{\mathbf{p}} a_{\mathbf{p} \uparrow}^{\dagger} a_{-\mathbf{p} \downarrow}^{\dagger} \sum_{\mathbf{p}^{\prime} \neq \mathbf{p}} a_{-\mathbf{p}^{\prime} \downarrow} a_{\mathbf{p}^{\prime} \uparrow}+N \uparrow N \downarrow\right)\right| \Psi_{2 \nu}\right\rangle \\
=\frac{U}{V}\left(\sum_{\mathbf{p}, \mathbf{p}^{\prime}} g_{\mathbf{p}} g_{\mathbf{p}^{\prime}} \sum_{s\left(\nu-1: \mathbf{p}, \mathbf{p}^{\prime}\right)} \prod_{i=1}^{\nu-1} g_{\mathbf{k}_{i}}^{2}+\nu^{2}\left\langle\Psi_{2 \nu} \mid \Psi_{2 \nu}\right\rangle\right) .
\end{gathered}
$$

Note well that the sum over the set $s\left(\nu-1: \mathbf{p}, \mathbf{p}^{\prime}\right)$ ensures $\mathbf{p} \neq \mathbf{p}^{\prime}$. Now define

$$
A_{n}^{m}\left(\mathbf{p}_{1}, \ldots \mathbf{p}_{m}\right) \equiv \sum_{s\left(n: \mathbf{p}_{1}, \mathbf{p}_{2}, \ldots \mathbf{p}_{m}\right)} \prod_{i=1}^{n} g_{\mathbf{k}_{i}}^{2},
$$

and

$$
B_{n}^{m}\left(\mathbf{p}_{1}, \ldots \mathbf{p}_{m}\right) \equiv \frac{A_{n}^{m}\left(\mathbf{p}_{1}, \ldots \mathbf{p}_{m}\right)}{A_{\nu}^{0}}
$$

which both vanish if any two momenta are equal, to express $E_{2 \nu}$ as

$$
\begin{aligned}
& E_{2 \nu}-\nu^{2} \frac{U}{V} \\
& =\frac{2 \sum_{\mathbf{p}} \epsilon_{\mathbf{p}} g_{\mathbf{p}}^{2} A_{\nu-1}^{1}(\mathbf{p})+\frac{U}{V} \sum_{\mathbf{p}, \mathbf{p}^{\prime}} g_{\mathbf{p}} g_{\mathbf{p}^{\prime}} A_{\nu-1}^{2}\left(\mathbf{p}, \mathbf{p}^{\prime}\right)}{A_{\nu}^{0}} \\
& =2 \sum_{\mathbf{p}} \epsilon_{\mathbf{p}} g_{\mathbf{p}}^{2} B_{\nu-1}^{1}(\mathbf{p})+\frac{U}{V} \sum_{\mathbf{p}, \mathbf{p}^{\prime}} g_{\mathbf{p}} g_{\mathbf{p}^{\prime}} B_{\nu-1}^{2}\left(\mathbf{p}, \mathbf{p}^{\prime}\right)
\end{aligned}
$$

Next, we evaluate the BCS variational equation for a state with $N_{e}=2 \nu$ electrons

$$
\frac{\partial E_{2 \nu}}{\partial g_{\mathbf{p}}}=0
$$

by first calculating

$$
\begin{aligned}
\frac{\partial A_{n}^{m}\left(\mathbf{p}_{1}, \ldots \mathbf{p}_{m}\right)}{\partial g_{\mathbf{p}}} & =2 g_{\mathbf{p}} \sum_{s\left(n-1: \mathbf{p}_{1}, \mathbf{p}_{2}, \ldots \mathbf{p}_{m}, \mathbf{p}\right)} \prod_{i=1}^{n-1} g_{\mathbf{k}_{i}}^{2} \\
& =2 g_{\mathbf{p}} A_{n-1}^{m+1}\left(\mathbf{p}_{1}, \ldots, \mathbf{p}_{m}, \mathbf{p}\right)
\end{aligned}
$$

Then we use the product rule for differentiating,

$$
\begin{aligned}
& A_{\nu}^{0} \frac{\partial B_{n}^{m}\left(\mathbf{p}_{1}, \ldots \mathbf{p}_{m}\right)}{\partial g_{\mathbf{p}}} \\
& =\frac{\partial A_{n}^{m}\left(\mathbf{p}_{1}, \ldots \mathbf{p}_{m}\right)}{\partial g_{\mathbf{p}}}-B_{n}^{m}\left(\mathbf{p}_{1}, \ldots \mathbf{p}_{m}\right) \frac{\partial A_{\nu}^{0}}{\partial g_{\mathbf{p}}} \\
& =2 g_{\mathbf{p}}\left(A_{n-1}^{m+1}\left(\mathbf{p}_{1}, \ldots \mathbf{p}_{m}, \mathbf{p}\right)-B_{n}^{m}\left(\mathbf{p}_{1}, \ldots \mathbf{p}_{m}\right) A_{\nu-1}^{1}(\mathbf{p})\right),
\end{aligned}
$$

to obtain

$$
\begin{aligned}
0 & =A_{\nu}^{0} \frac{\partial E_{2 \nu}}{\partial g_{\mathbf{p}}} \\
& =4 \epsilon_{\mathbf{p}} g_{\mathbf{p}} A_{\nu-1}^{1}(\mathbf{p})+4 \sum_{\mathbf{p}^{\prime}} \epsilon_{\mathbf{p}^{\prime}} g_{\mathbf{p}^{\prime}}^{2} g_{\mathbf{p}} A_{\nu-2}^{2}\left(\mathbf{p}^{\prime}, \mathbf{p}\right)
\end{aligned}
$$

$$
\begin{aligned}
& +\frac{2 U}{V} \sum_{\mathbf{p}^{\prime}} g_{\mathbf{p}^{\prime}} A_{\nu-1}^{2}\left(\mathbf{p}^{\prime}, \mathbf{p}\right) \\
& +\frac{2 U}{V} \sum_{\mathbf{p}^{\prime}, \mathbf{p}^{\prime \prime}} g_{\mathbf{p}^{\prime}} g_{\mathbf{p}^{\prime \prime}} g_{\mathbf{p}} A_{\nu-2}^{3}\left(\mathbf{p}^{\prime}, \mathbf{p}^{\prime \prime}, \mathbf{p}\right) \\
& -2 g_{\mathbf{p}} A_{\nu-1}^{1}(\mathbf{p})\left(E_{2 \nu}-\frac{\nu^{2} U}{V}\right)
\end{aligned}
$$

Solving for $g_{\mathbf{p}}$ yields

$$
g_{\mathbf{p}}=\frac{U}{V \mathcal{D}_{e}} \sum_{\mathbf{p}^{\prime}} g_{\mathbf{p}^{\prime}} A_{\nu-1}^{2}\left(\mathbf{p}^{\prime}, \mathbf{p}\right)
$$

with the denominator,

$$
\begin{aligned}
\mathcal{D}_{e} & =A_{\nu-1}^{1}(\mathbf{p})\left(E_{2 \nu}-\frac{\nu^{2} U}{V}-2 \epsilon_{\mathbf{p}}\right) \\
& -2 \sum_{\mathbf{p}^{\prime}} \epsilon_{\mathbf{p}^{\prime}} g_{\mathbf{p}^{\prime}}^{2} A_{\nu-2}^{2}\left(\mathbf{p}^{\prime}, \mathbf{p}\right) \\
& -\frac{U}{V} \sum_{\mathbf{p}^{\prime}, \mathbf{p}^{\prime \prime}} g_{\mathbf{p}^{\prime}} g_{\mathbf{p}^{\prime \prime}} A_{\nu-2}^{3}\left(\mathbf{p}^{\prime}, \mathbf{p}^{\prime \prime}, \mathbf{p}\right) .
\end{aligned}
$$

Equation (17) is equivalent to Eq. (34) of Ref. 8 (note their equation has a misprint involving a missing $g_{\mathbf{p}}$ ).

In the case of odd filling, $N_{e}=2 \nu+1$, the variational state has a single specific unpaired, say up, electron with momentum q, chosen to minimize the energy:

$$
\left|\Psi_{2 \nu+1}\right\rangle=\sum_{s(\nu: \mathbf{q})}\left(\prod_{i=1}^{\nu} g_{\mathbf{k}_{i}} a_{\mathbf{k}_{i} \uparrow}^{\dagger} a_{-\mathbf{k}_{i} \downarrow}^{\dagger}\right) a_{\mathbf{q} \uparrow}^{\dagger}|0\rangle
$$

Operating with the Hamiltonian on this state shifts the energy by $\epsilon_{\mathbf{q} \uparrow}+\frac{U}{V} N_{\downarrow}$ and repeating the above analysis yields

$$
\begin{aligned}
& E_{2 \nu+1}-\epsilon_{\mathbf{q}}-\nu(\nu+1) \frac{U}{V} \\
& =\frac{2 \sum_{\mathbf{p}} \epsilon_{\mathbf{p}} g_{\mathbf{p}}^{2} A_{\nu-1}^{2}(\mathbf{p}, \mathbf{q})+\frac{U}{V} \sum_{\mathbf{p}, \mathbf{p}^{\prime}} g_{\mathbf{p}} g_{\mathbf{p}^{\prime}} A_{\nu-1}^{3}\left(\mathbf{p}, \mathbf{p}^{\prime}, \mathbf{q}\right)}{A_{\nu}^{1}(\mathbf{q})} \\
& =2 \sum_{\mathbf{p}} \epsilon_{\mathbf{p}} g_{\mathbf{p}}^{2} C_{\nu-1}^{1}(\mathbf{p})+\frac{U}{V} \sum_{\mathbf{p}, \mathbf{p}^{\prime}} g_{\mathbf{p}} g_{\mathbf{p}^{\prime}} C_{\nu-1}^{2}\left(\mathbf{p}, \mathbf{p}^{\prime}\right)
\end{aligned}
$$

where

$$
C_{n}^{m}\left(\mathbf{p}_{1}, \ldots \mathbf{p}_{m}\right) \equiv \frac{A_{n}^{m+1}\left(\mathbf{p}_{1}, \ldots \mathbf{p}_{m}, \mathbf{q}\right)}{A_{\nu}^{1}(\mathbf{q})}
$$

is equivalent to $B_{n}^{m}\left(\mathbf{p}_{1}, \ldots \mathbf{p}_{m}\right)$ defined on a space with the $\mathbf{q}$ mode removed. Finally, the odd electron system variational equation reduces to

$$
g_{\mathbf{p}}=\frac{U}{V \mathcal{D}_{o}} \sum_{\mathbf{p}^{\prime}} g_{\mathbf{p}^{\prime}} A_{\nu-1}^{3}\left(\mathbf{p}^{\prime}, \mathbf{p}, \mathbf{q}\right)
$$

where 


$$
\begin{aligned}
\mathcal{D}_{o} & =A_{\nu-1}^{2}(\mathbf{p}, \mathbf{q})\left(E_{2 \nu+1}-\frac{\nu(\nu+1) U}{V}-2 \epsilon_{\mathbf{p}}-\epsilon_{\mathbf{q}}\right) \\
& -2 \sum_{\mathbf{p}^{\prime}} \epsilon_{\mathbf{p}^{\prime}} g_{\mathbf{p}^{\prime}}^{2} A_{\nu-2}^{3}\left(\mathbf{p}^{\prime}, \mathbf{p}, \mathbf{q}\right) \\
& -\frac{U}{V} \sum_{\mathbf{p}^{\prime}, \mathbf{p}^{\prime \prime}} g_{\mathbf{p}^{\prime}} g_{\mathbf{p}^{\prime \prime}} A_{\nu-2}^{4}\left(\mathbf{p}^{\prime}, \mathbf{p}^{\prime \prime}, \mathbf{p}, \mathbf{q}\right)
\end{aligned}
$$

\section{Exact diagonalization}

Using PARPACK, a parallel implementation of the Lanczos algorithm, we exactly calculated the Hubbard and reduced Hamiltonian ground states as a function of the electron density. The dimension of the Hilbert space for a 16 site Hubbard model is $4^{16} \approx 4 \times 10^{9}$. Since the Hamiltonians conserve momentum and particle number we may restrict to subspaces with fixed momentum and filling. However, the dimension for half-filling at $0 \mathrm{mo}-$ mentum is still greater than $10^{7}$. There are about 2000 non-zero terms for each state, so the Hubbard Hamiltonian matrix cannot be stored in memory even in a sparse format. Instead, matrix elements were generated at each step in the Lanczos algorithm. The diagonalization took about 3 hours using $301.2 \mathrm{Ghz}$ Athlon processors in parallel.

For even $N_{e}=2 \nu$, we restrict to the zero momentum subspace which yields the minimum energy eigenvector. For odd $N_{e}$, the momentum with lowest ground state energy is dependent on the coupling $U$. For $U=0$ it is obvious that the lowest energy will have the momentum of the $\nu+1$ th lowest energy mode. For high $U$, with less than half filling, the lowest energy will have 0 momentum, but for the value $U=-10$ used throughout this paper, the momentum is the same as for $U=0$.

The total momentum of the ground state for odd $N_{e}$ corresponds to occupation of a specific unpaired momentum mode. For the Hubbard model, occupation of this mode is not fixed by the action of the Hamiltonian but, for the reduced model, all relevant basis states have the same unpaired mode. In this regard, the reduced model ground state is the same as the BCS state which has the single unpaired mode $\mathbf{q}$ (Eq. [19).

\section{Observables and theoretical constructs}

To properly assess any approximation scheme, as well as investigate the robustness of a particular dynamic model, it is important to calculate and compare a variety of observables and theoretical constructs.

In addition to the ground state energy, $E$, and energy density, $E / V$, another important observable is the single particle energy gap, $\Delta_{N_{e}}$,

$$
\Delta_{N_{e}}=\frac{1}{2}\left[E_{N_{e}+1}-E_{N_{e}}+E_{N_{e}-1}-E_{N_{e}}\right]
$$

$$
=\frac{1}{2}\left[E_{N_{e}+1}-2 E_{N_{e}}+E_{N_{e}-1}\right] .
$$

In the grand canonical formulation, a quasi-particle excitation is produced by the action of $a_{\mathbf{p} \uparrow}^{\dagger}$, or equivalently, $a_{-\mathbf{p} \downarrow}$ on the state $|\mathrm{BCS}\rangle{ }^{11}$ with excitation energy

$$
\begin{aligned}
E_{\mathbf{p}} & \equiv \frac{\left\langle\mathrm{BCS}\left|a_{\mathbf{p} \uparrow} H a_{\mathbf{p} \uparrow}^{\dagger}\right| \mathrm{BCS}\right\rangle}{\langle B C S \mid B C S\rangle}-\frac{\langle\mathrm{BCS}|H| \mathrm{BCS}\rangle}{\langle B C S \mid B C S\rangle} \\
& =\sqrt{\Delta_{\mathrm{BCS}}^{2}+\epsilon_{\mathbf{p}}^{2}} .
\end{aligned}
$$

Here $\Delta_{\mathrm{BCS}}$ is the BCS gap and $\epsilon_{\mathbf{p}}$ is dependent on the momentum p. The minimum excitation is non-zero since $E_{\mathbf{p}} \geq \Delta_{\text {BCS }}$, thus accounting for the name "gap". In the canonical BCS formulation $a_{\mathbf{p} \uparrow}^{\dagger} \Psi_{2 \nu}$ is no longer proportional to $a_{\mathbf{p} \downarrow} \Psi_{2 \nu}$. Therefore, $\Delta_{N_{e}}$ averages the effects of adding and subtracting one electron.

Because wavefunctions are more sensitive than energies to approximation schemes, we also address other operator wavefunction overlaps such as propagators and correlators 9 . Before introducing theses constructs we first specify the generalized one, $\rho_{\sigma \tau}(r, s)$, and two, $\rho_{\sigma \sigma^{\prime} \tau \tau^{\prime}}\left(\mathbf{r}, \mathbf{r}^{\prime}, \mathbf{s}, \mathbf{s}^{\prime}\right)$, particle density matrices for any model state $|\Psi\rangle$ with unit norm

$$
\begin{gathered}
\rho_{\sigma \tau}(\mathbf{r}, \mathbf{s})=\left\langle\Psi\left|c_{\mathbf{r} \sigma}^{\dagger} c_{\mathbf{s} \tau}\right| \Psi\right\rangle \equiv\left\langle c_{\mathbf{r} \sigma}^{\dagger} c_{\mathbf{s} \tau}\right\rangle \\
\rho_{\sigma \sigma^{\prime} \tau \tau^{\prime}}\left(\mathbf{r}, \mathbf{r}^{\prime}, \mathbf{s}, \mathbf{s}^{\prime}\right)=\left\langle c_{\mathbf{r} \sigma}^{\dagger} c_{\mathbf{r}^{\prime} \sigma^{\prime}}^{\dagger} c_{\mathbf{s} \tau} c_{\mathbf{s}^{\prime} \tau^{\prime}}\right\rangle .
\end{gathered}
$$

Here the indices $\mathbf{r}, \mathbf{s}$ represent possible electron lattice sites. The position operators, $c_{\mathbf{r} \sigma}$ and $c_{\mathbf{r} \sigma}^{\dagger}$, are related to the momentum operators by

$$
\begin{aligned}
& c_{\mathbf{r}}=\frac{1}{\sqrt{V}} \sum_{\mathbf{p}} e^{-i \mathbf{p} \cdot \mathbf{r}} a_{\mathbf{p}} \\
& c_{\mathbf{r}}^{\dagger}=\frac{1}{\sqrt{V}} \sum_{\mathbf{p}} e^{i \mathbf{p} \cdot \mathbf{r}} a_{\mathbf{p}}^{\dagger} .
\end{aligned}
$$

Between states with the same filling $\rho_{\uparrow \downarrow}(\mathbf{r}, \mathbf{s})=$ $\rho_{\downarrow \uparrow}(\mathbf{r}, \mathbf{s})=0$, while $\rho_{\uparrow \uparrow}(\mathbf{r}, \mathbf{s})$ and $\rho_{\downarrow \downarrow}(\mathbf{r}, \mathbf{s})$ are the one electron propagators for spin up or down, respectively.

Next we construct a variety of correlators as the difference between a specific two particle density and the product of one particle densities. For example, the on site $s$-wave correlator, $C_{s}(\mathbf{r}, \mathbf{s})$, is

$$
C_{s}(\mathbf{r}, \mathbf{s})=\rho_{\uparrow \downarrow \downarrow \uparrow}(\mathbf{r}, \mathbf{r}, \mathbf{s}, \mathbf{s})-\rho_{\uparrow \uparrow}(\mathbf{r}, \mathbf{s}) \rho_{\downarrow \downarrow}(\mathbf{r}, \mathbf{s}),
$$

which is the correlated electron pair propagator minus the product of two (independent) one electron propagators. The extended $s$-wave correlator, $C_{e s}(\mathbf{r}, \mathbf{s})$, is

$$
\begin{aligned}
C_{e s}(\mathbf{r}, \mathbf{s})=\sum_{\mathbf{t}, \mathbf{t}^{\prime}} & w_{e s}(\mathbf{t}) w_{e s}\left(\mathbf{t}^{\prime}\right)\left(\rho_{\uparrow \downarrow \downarrow \uparrow}\left(\mathbf{r}, \mathbf{r}+\mathbf{t}, \mathbf{s}, \mathbf{s}+\mathbf{t}^{\prime}\right)\right. \\
& \left.-\rho_{\uparrow \uparrow}(\mathbf{r}, \mathbf{s}) \rho_{\downarrow \downarrow}\left(\mathbf{r}+\mathbf{t}, \mathbf{s}+\mathbf{t}^{\prime}\right)\right),
\end{aligned}
$$


which involves a sum, $\mathbf{t}=\left(t_{x}, t_{y}\right), \mathbf{t}^{\prime}=\left(t_{x}^{\prime}, t_{y}^{\prime}\right)$, over the four planar sites adjacent to lattice site $\mathbf{r}(\mathbf{s})$ with weights $w_{e s}(\mathbf{t}), w_{e s}\left(\mathbf{t}^{\prime}\right)$ given by $w_{e s}(\mathbf{t})=1$ for $\mathbf{t}=$ $(1,0),(0,1),(-1,0)$ or $(0,-1)$ and $w_{e s}(\mathbf{t})=0$ otherwise.

We also list in Table I several other correlators, $C_{\text {sym }}$, with different symmetry weights $w_{\text {sym }}(\mathbf{t})$ which are important for investigating superconductivity. See Ref. $\underline{9}$ for illustrations and further discussion.

TABLE I: Correlators

\begin{tabular}{|c|c|c|}
\hline Correlator & $C_{s y m}$ & non-zero $w_{\text {sym }}(\mathbf{t})$ terms \\
\hline \hline$s$-wave & $C_{s}$ & $w_{s}(0,0)=1$ \\
\hline $\begin{array}{c}\text { extended } \\
s \text {-wave }\end{array}$ & $C_{e s}$ & $\begin{array}{c}w_{e s}(1,0)=w_{e s}(-1,0)=1 \\
w_{e s}(0,1)=w_{e s}(0,-1)=1\end{array}$ \\
\hline $\begin{array}{c}x \text { direction } \\
p \text {-wave }\end{array}$ & $C_{p_{x}}$ & $\begin{array}{c}w_{p_{x}}(1,0)=1 \\
w_{p_{x}}(-1,0)=-1\end{array}$ \\
\hline$d$-wave & $C_{d_{x^{2}-y^{2}}}$ & $\begin{array}{c}w_{d_{x^{2}-y^{2}}}(1,0)=w_{d_{x^{2}-y^{2}}}(-1,0)=1 \\
w_{d_{x^{2}-y^{2}}}(0,1)=w_{x_{x^{2}-y^{2}}}(0,-1)=-1\end{array}$ \\
\hline $\begin{array}{c}\text { diagonal } \\
d \text {-wave }\end{array}$ & $C_{d_{x y}}$ & $\begin{array}{c}w_{d_{x y}}(1,1)=w_{d_{x y}}(-1,-1)=1 \\
w_{d_{x y}}(1,-1)=w_{d_{x y}}(-1,1)=-1\end{array}$ \\
\hline
\end{tabular}

Since our system has translational symmetry, the correlator, $C_{\text {sym }}(\mathbf{r}, \mathbf{r}+\mathbf{d})$, and propagator, $\rho_{\sigma \sigma}(\mathbf{r}, \mathbf{r}+\mathbf{d})$, do not depend on $\mathbf{r}$. Therefore we can write

$$
\begin{gathered}
C_{\text {sym }}(\mathbf{d}) \equiv C_{\text {sym }}(\mathbf{r}, \mathbf{r}+\mathbf{d})=\frac{1}{V} \sum_{\mathbf{s}} C_{\text {sym }}(\mathbf{s}, \mathbf{s}+\mathbf{d}) \\
\rho_{\sigma \sigma}(\mathbf{d}) \equiv \rho_{\sigma \sigma}(\mathbf{r}, \mathbf{r}+\mathbf{d})=\frac{1}{V} \sum_{\mathbf{s}} \rho_{\sigma \sigma}(\mathbf{s}, \mathbf{s}+\mathbf{d}) .
\end{gathered}
$$

As discussed in Ref. 9, an effective measure of superconductivity is the concept of Off-Diagonal Long Range Order (ODLRO) which can be quantitatively characterized by the correlator or ODLRO function, $C_{\text {sym }}(\mathbf{d})$. The criterion for demonstrating superconductivity is that $C_{\text {sym }}(\mathbf{d})$ remains finite, positive and does not decrease to zero at large $\mathbf{r}$.

Using Eqs. (28 29), the propagator can be written in momentum space as

$$
\begin{aligned}
\rho_{\uparrow \uparrow}(\mathbf{d}) & =\frac{1}{V} \sum_{\mathbf{r}}\left\langle c_{\mathbf{r} \uparrow}^{\dagger} c_{\mathbf{r}+\mathbf{d} \uparrow}\right\rangle \\
& =\frac{1}{V^{2}} \sum_{\mathbf{p}, \mathbf{q}} P(\mathbf{p}, \mathbf{q}, \mathbf{d})\left\langle a_{\mathbf{p} \uparrow}^{\dagger} a_{\mathbf{q} \uparrow}\right\rangle,
\end{aligned}
$$

where

$$
\begin{aligned}
P(\mathbf{p}, \mathbf{q}, \mathbf{d}) & =\sum_{r} e^{i(\mathbf{p} \cdot \mathbf{r}-\mathbf{q} \cdot(\mathbf{r}+\mathbf{d}))} \\
& =V \delta_{\mathbf{p q}} e^{-i \mathbf{q} \cdot \mathbf{d}}
\end{aligned}
$$

Thus

$$
\rho_{\uparrow \uparrow}(\mathbf{d})=\frac{1}{V} \sum_{\mathbf{p}} e^{-i \mathbf{p} \cdot \mathbf{d}}\left\langle a_{\mathbf{p} \uparrow}^{\dagger} a_{\mathbf{p} \uparrow}\right\rangle,
$$

and evaluating $C_{\text {sym }}(\mathbf{d})$ in momentum space yields

$$
\begin{aligned}
& C_{\text {sym }}(\mathbf{d})=\frac{1}{V} \sum_{\mathbf{r}, \mathbf{t}, \mathbf{t}^{\prime}} w_{\text {sym }}(\mathbf{t}) w_{\text {sym }}\left(\mathbf{t}^{\prime}\right) \\
& \rho_{\uparrow \downarrow \downarrow \uparrow}\left(\mathbf{r}, \mathbf{r}+\mathbf{t}, \mathbf{r}+\mathbf{d}+\mathbf{t}^{\prime}, \mathbf{r}+\mathbf{d}\right) \\
& =\frac{1}{V^{3}} \sum_{\mathbf{p}, \mathbf{p}^{\prime}, \mathbf{p}^{\prime \prime}, \mathbf{p}^{\prime \prime \prime}} P\left(\mathbf{p}, \mathbf{p}^{\prime}, \mathbf{p}^{\prime \prime}, \mathbf{p}^{\prime \prime \prime}, \mathbf{d}\right)\left\langle a_{\mathbf{p} \uparrow}^{\dagger} a_{\mathbf{p}^{\prime} \downarrow}^{\dagger} a_{\mathbf{p}^{\prime \prime} \downarrow} a_{\mathbf{p}^{\prime \prime \prime} \uparrow}\right\rangle \\
& P\left(\mathbf{p}, \mathbf{p}^{\prime}, \mathbf{p}^{\prime \prime}, \mathbf{p}^{\prime \prime \prime}, \mathbf{d}\right)=V \delta_{\mathbf{p}, \mathbf{p}^{\prime \prime}+\mathbf{p}^{\prime \prime \prime}-\mathbf{p}^{\prime}} e^{-i \mathbf{d} \cdot\left(\mathbf{p}^{\prime \prime}+\mathbf{p}^{\prime \prime \prime}\right)} \\
& \sum_{\mathbf{t}} w_{\text {sym }}(\mathbf{t}) e^{i \mathbf{p}^{\prime} \cdot \mathbf{t}} \sum_{\mathbf{t}^{\prime}} w_{\text {sym }}\left(\mathbf{t}^{\prime}\right) e^{-i \mathbf{p}^{\prime \prime} \cdot \mathbf{t}^{\prime}} \text {. }
\end{aligned}
$$

In particular for d-wave symmetry

$$
\begin{aligned}
& P\left(\mathbf{p}, \mathbf{p}^{\prime}, \mathbf{p}^{\prime \prime}, \mathbf{p}^{\prime \prime \prime}, \mathbf{d}\right)=4 V \delta_{\mathbf{p}, \mathbf{p}^{\prime \prime}+\mathbf{p}^{\prime \prime \prime}-\mathbf{p}^{\prime}} e^{-i \mathbf{d} \cdot\left(\mathbf{p}^{\prime \prime}+\mathbf{p}^{\prime \prime \prime}\right)} \\
& \left(\cos \left(p_{x}^{\prime}\right)-\cos \left(p_{y}^{\prime}\right)\right)\left(\cos \left(p_{x}^{\prime \prime}\right)-\cos \left(p_{y}^{\prime \prime}\right)\right) .
\end{aligned}
$$

The other correlators were calculated similarly.

\section{NUMERICAL RESULTS AND DISCUSSION}

\section{A. One dimensional systems}

We first compute for a one dimensional lattice the approximate BCS and exact Hubbard and reduced Hamiltonians ground state energies as a function of level filling. In Fig. 1 the Hubbard (boxes), $E / V$, reduced (circles), $E_{r} / V$, and BCS (crosses), $E_{B C S} / V$, ground state energy densities are compared as a function of electron filling, $N_{e}$, for $t=1, V=16$ sites and $U=-10$. The BCS and exact Hubbard data reproduce the data in Refs. 6 and 8 . As they reported, the agreement is very good, especially for the case of even filling. The exact reduced data is indistinguishable from the BCS data in the figure. Over $99 \%$ of the difference between the BCS and the exact Hubbard is due to the difference between $H_{r}$ and $H$ and only $1 \%$ is due to quantum correlations between different BCS pairs which are excluded by the variational wavefunction. Similar results have been obtained for other potential strengths and different number of lattice sites (not shown).

The Hubbard (boxes), reduced (circles) and BCS (crosses) energy gaps, $\Delta_{N_{e}}$, are compared in Fig. 2] As with the ground state energy, the BCS gap is the same for either the Hubbard or reduced Hamiltonian. In contrast to energy density comparisons, the BCS-Hubbard difference is more pronounced especially for half-filling, $N_{e}=V$. The BCS is still a good treatment for the reduced Hamiltonian.

\section{B. Two dimensional systems}

Having reproduced and extended one dimensional studies, we now address two dimensional systems. Figure 


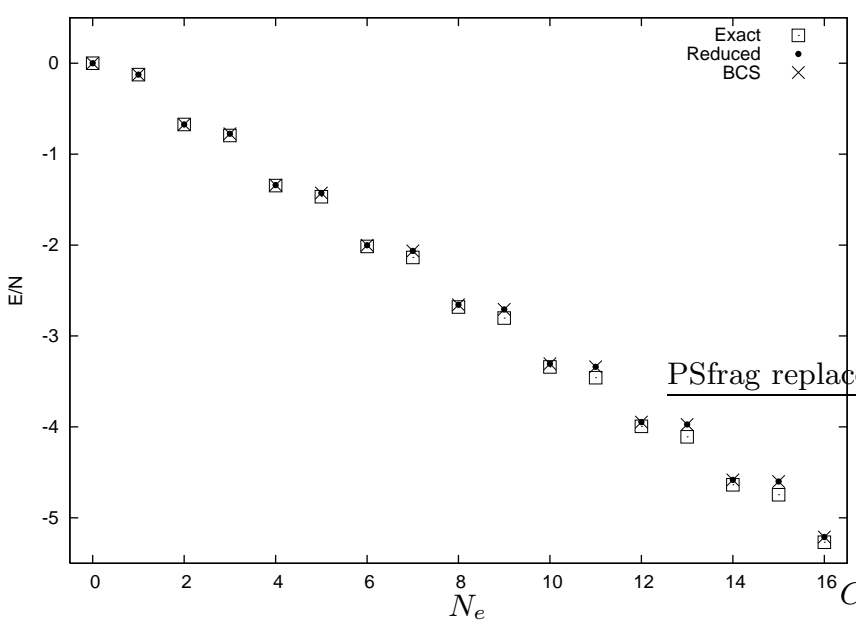

FIG. 1: One dimensional ground state energy densities as a function of electron filling for the Hubbard (boxes) and reduced (circles) Hamiltonians. Note the good agreement with the BCS results (crosses).

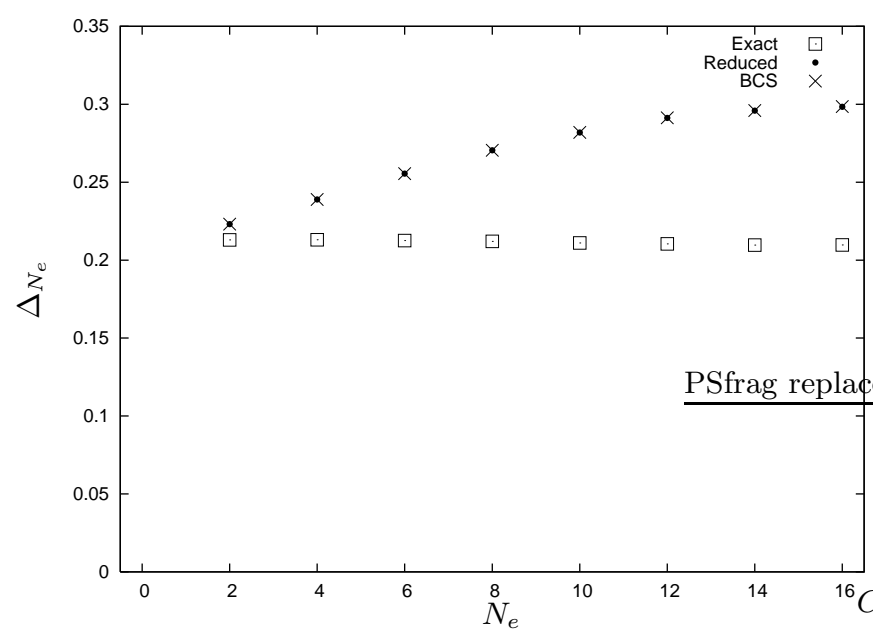

FIG. 2: One dimensional energy gaps as a function of electron filling for the Hubbard (boxes) and reduced (circles) Hamiltonians. The BCS (crosses) is least accurate near half-filling.

3 depicts the Hubbard, reduced and BCS energy densities for a two dimensional lattice having $V=4 \times 4=16$ sites.

Again the BCS energies are close to the exact Hubbard eigenvalues and indistinguishable from the reduced eigenvalues. The corresponding energy gap comparisons are displayed in Fig. 4. Contrary to conjecture, the difference between the exact and BCS energy gaps remains similar to the one dimensional case.

We further note that the Hubbard and reduced energy eigenvalues are generally in reasonably good agreement for all electron densities in both one and two dimensions. Accordingly, it would appear that the reduced Hamilto-

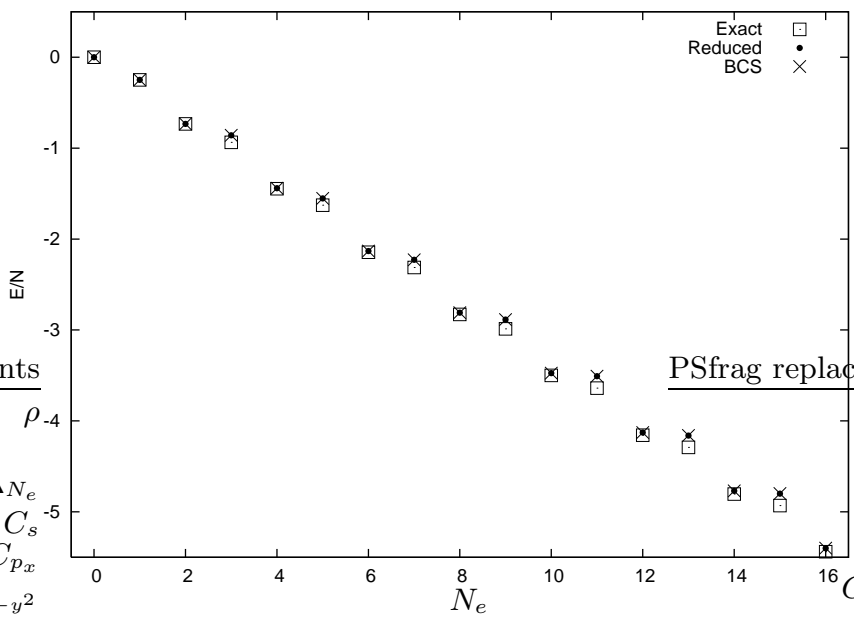

FIG. 3: Two dimensional ground state energy densities as a function of electron filling for the Hubbard (boxes) and reduced (circles) Hamiltonians. Note the good agreement with the BCS results (crosses).

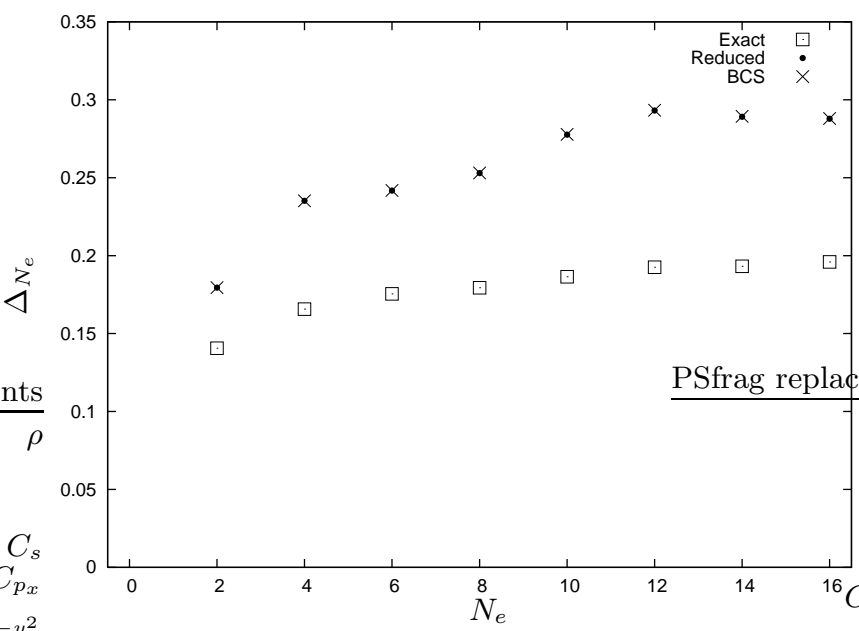

FIG. 4: Two dimensional energy gaps as a function of electron filling for the exact Hubbard (boxes) and reduced (circles) Hamiltonians. Note the disagreement between the BCS (crosses) and the Hubbard energy gaps, similar to the one dimensional result (compare to Fig. 2).

nian incorporates the dominant physical dynamics. This is fortuitous because $H_{r}$ can be solved more easily than $H$, whether by the BCS approximation or by diagonalization, which in turn facilitates investigating more complex systems in multi-dimensions.

\section{Wavefunction overlap}

In general, a variational calculation reproduces the ground state energy much better than other observables. It is therefore of interest to examine the overlap of the 
BCS and exact model wavefunctions. Figure 5 plots the inner product of the exact, one dimensional Hubbard eigenvector with the BCS state as a function of electron filling. Notice the significant deviation from unity especially with increasing density. As before, the BCS and Hubbard results have the largest difference for systems near half-filling and odd number of electrons. In two dimensions the overlap behavior is similar but now the values are significantly higher (see Fig. 6), consistent with the conjecture that the BCS approximation improves with higher dimensionality. The inner product of the reduced model eigenvector with the $|B C S\rangle$ is .999 in both 1 and 2 dimensions (not shown).

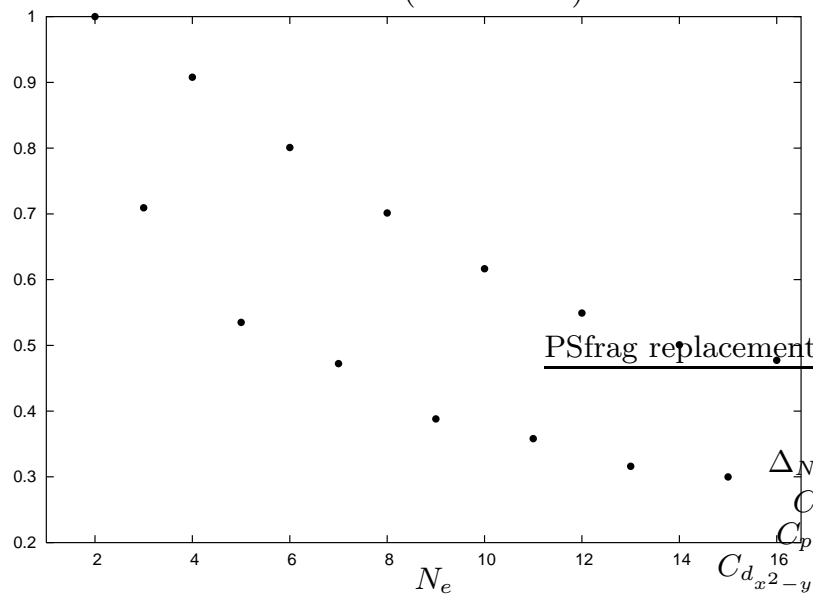

FIG. 5: Inner product of the Hubbard and BCS vectors as a function of filling $N_{e}$ (one dimension).

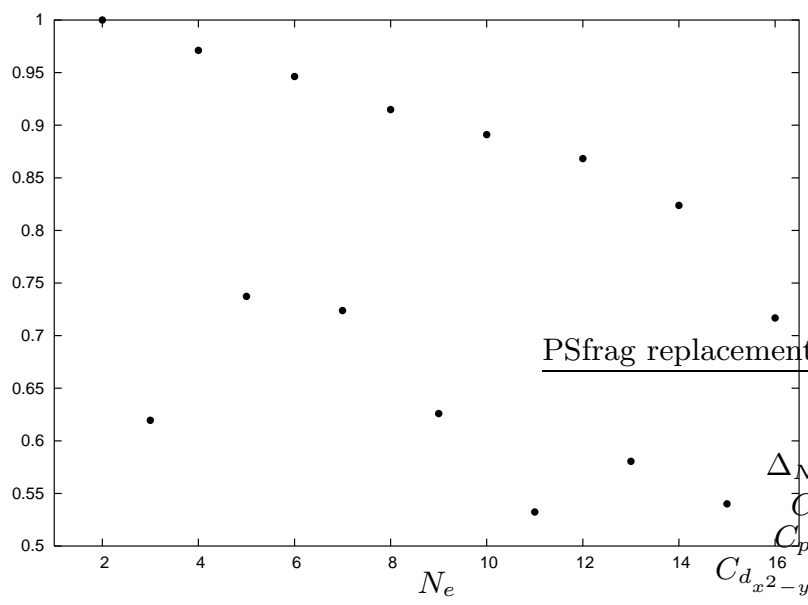

FIG. 6: Inner product of the Hubbard and BCS vectors as a function of filling $N_{e}$ (two dimensions).

\section{Densities, propagators and correlators}

Returning to physical observables, this section compares propagators and correlators for exact and approximate model wavefunctions. Unless stated otherwise, all calculations were performed for $U=-10$, lattice sizes $V=16 \times 1$ and $V=4 \times 4$, and $N_{e}=15$ and $N_{e}=16$.
Results for other fillings were also calculated and found to be qualitatively similar.

Figures 7 and 8 compare the Hubbard, reduced and BCS electron propagators, $\rho_{\uparrow \uparrow}(d)$, for different lattice ranges $d$ and $N_{e}=16$ in one and two dimensions, respectively. The BCS and reduced propagators are essentially identical and also similar to the Hubbard result. At lower densities there are somewhat larger differences between the Hubbard and BCS (or reduced) propagators.

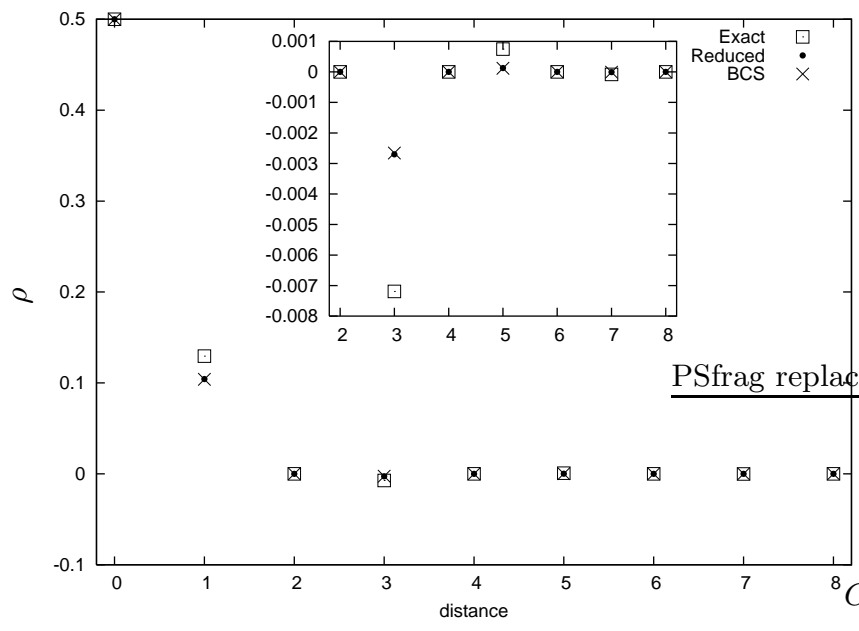

FIG. 7: One electron propagators as a function of distance (one dimension, $N_{e}=16$ ).

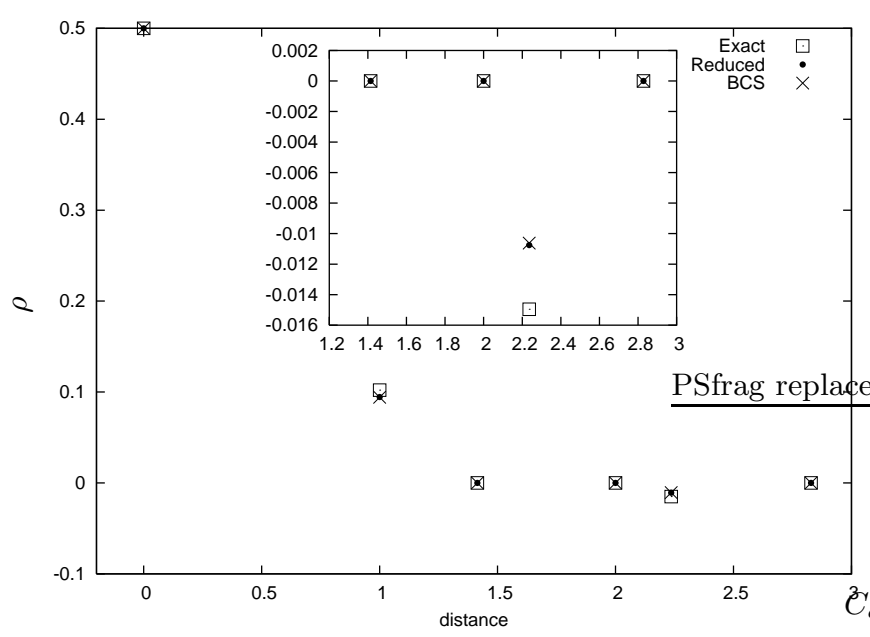

FIG. 8: One electron propagators as a function of distance for (two dimensions, $N_{e}=16$ ).

Figures 9 and [0] compare the different model single electron propagators again in one and two dimensions, respectively, but now $N_{e}=15$. Notice that, just like the model energy comparisons, the Hubbard and reduced propagators are more different for odd $N_{e}$, but again the BCS accurately reproduces the reduced results. Although lower $N_{e}$ Hubbard-reduced comparisons yield even larger devations (not shown), overall there is no significant difference in agreement between the two propagators in both one and two dimensions. 


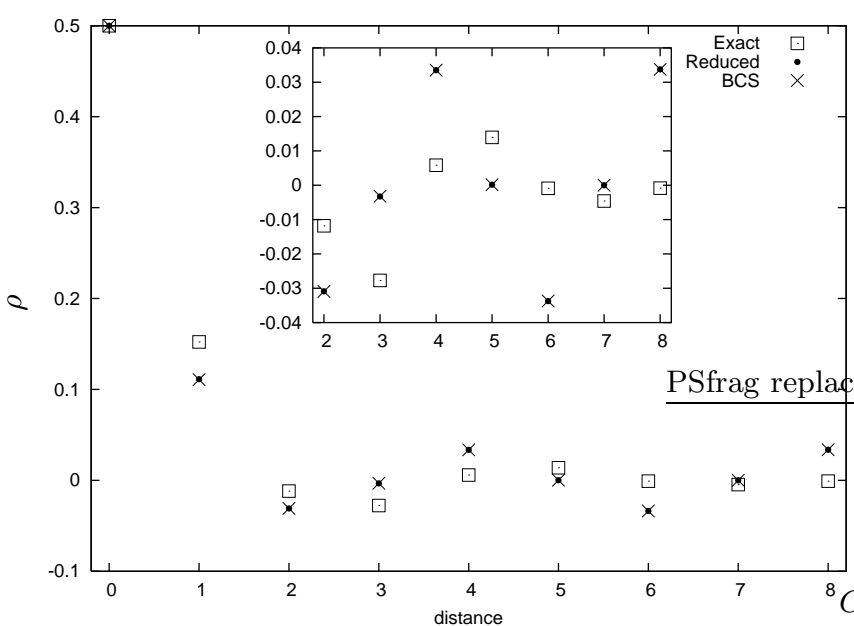

FIG. 9: One electron propagators as a function of distance (one dimension, $N_{e}=15$ ).

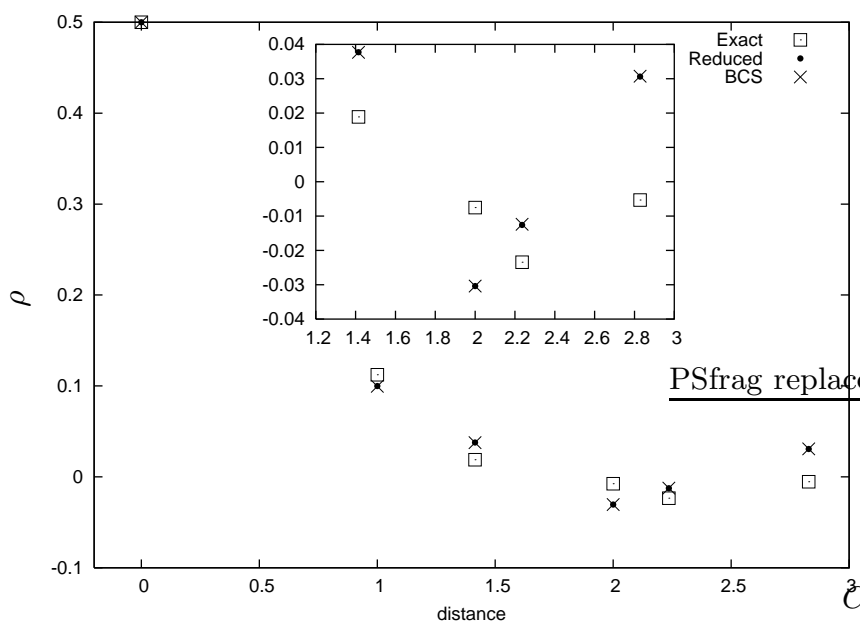

FIG. 10: One electron propagators as a function of distance (two dimensions, $N_{e}=15$ ).

Figures 11] and 12 similarly compare the Hubbard, reduced and BCS electron $s$-wave correlators, $C_{s}(d)$, for $N_{e}=16$ in one and two dimensions, respectively. Now there are significant departures between the Hubbard and reduced results. Notice that the reduced correlator has a larger magnitude than the Hubbard value. This is consistent with the energy gap comparison, where the reduced gap was larger than the Hubbard gap. Also, but not shown, lower $N_{e}$ yields smaller Hubbard-reduced correlator differences. The BCS again very accurately reproduces the reduced correlators.

The Hubbard and reduced $s$-wave correlators are closer in two dimensions than in one dimension but the limited range of $d$ sampled warrants caution in this conclusion. By the Mermin-Wagner theorem ${ }^{12}$, the Hubbard model will not display long range correlations at $T \neq 0$ in one or two dimensions. However, according to the Bethe ansatz $z^{13}$, at least for large $|U|$, the ground state energy of the one-dimensional system becomes part of a continuous spectrum as the system length increases. The theorem does not apply at $T=0$ under these circumstances and therefore long distance correlations are possible. The limited data shown in figures 11] and 12 are inconclusive on the question of long distance order.

Conversely, the BCS and reduced ODLRO functions are flat for increasing $d$. The Mermin-Wagner theorem does not apply to the reduced Hamiltonian, even at $T \neq$ 0 , because it has long range interactions. For the ground state of the reduced Hamiltonian, the $s$-wave correlator reduces to

$$
\begin{array}{r}
\frac{1}{V^{2}}\left(\sum_{\mathbf{p}, \mathbf{p}^{\prime}}\left\langle a_{\mathbf{p} \uparrow}^{\dagger} a_{-\mathbf{p} \downarrow}^{\dagger} a_{-\mathbf{p}^{\prime} \downarrow} a_{\mathbf{p}^{\prime} \uparrow}\right\rangle\right. \\
\left.+\sum_{\mathbf{p} \neq \mathbf{p}^{\prime}} e^{-i \mathbf{d} \cdot\left(\mathbf{p}-\mathbf{p}^{\prime}\right)}\left\langle a_{\mathbf{p}^{\dagger} \uparrow}^{\dagger} a_{-\mathbf{p}^{\prime} \downarrow}^{\dagger} a_{-\mathbf{p}^{\prime} \downarrow} a_{\mathbf{p} \uparrow}\right\rangle\right) .
\end{array}
$$

The second term decreases linearly with $V$ for large d, but the first term is independent of $\mathbf{d}$ and approaches a constant as $V$ increases. For $U, N_{e}, V$ and $|d| \gg 1$, this constant is $\frac{N_{e}\left(2 V-N_{e}\right)}{4 V^{2}}$. A precise limit can also be given for the BCS correlator at finite $V$. Resummation of the correlator yields terms independent of $\mathbf{d}$,

$$
\sum_{\mathbf{p} \neq \mathbf{p}^{\prime}} g_{\mathbf{p}} g_{\mathbf{p}^{\prime}} B_{\nu-1}^{2}\left(\mathbf{p}, \mathbf{p}^{\prime}\right)-\sum_{b=1}^{\nu}(-1)^{b} \sum_{\mathbf{p}} g_{\mathbf{p}}^{2 b} B_{\nu-b}^{1}(\mathbf{p}),
$$

with additional terms decreasing rapidly to 0 with increasing $\mathbf{d}$.

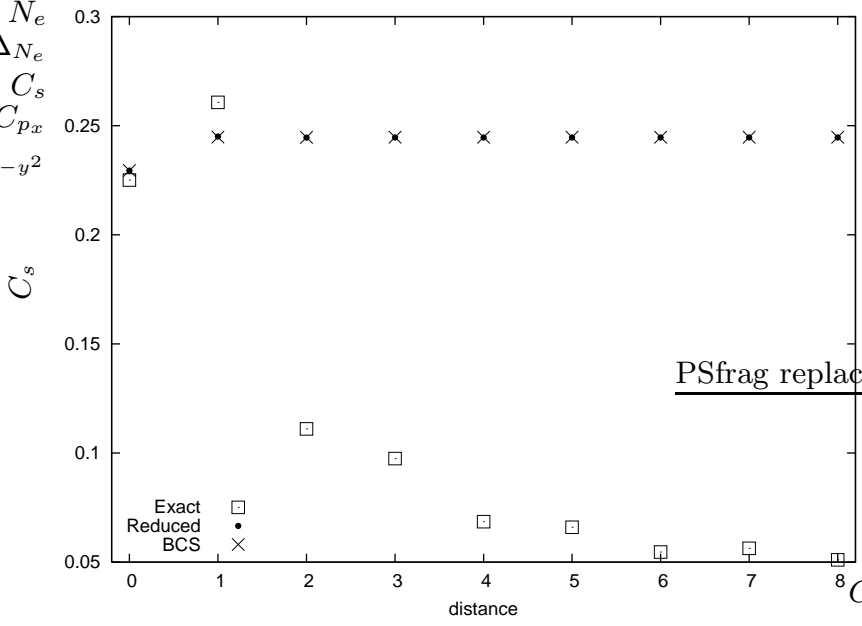

FIG. 11: The $s$-wave correlators as a function of distance (one dimension, $\left.N_{e}=16\right)$.

The Hubbard, reduced and BCS electron $s$-wave correlators, $C_{s}(d)$, for $N_{e}=15$ are compared in Figs. 13 and 14 for one and two dimensions, respectively. The differences are more dramatic for odd fillings as the Hubbard correlator in one dimension falls rapidly to 0 . The two dimensional Hubbard correlator also falls rapidly within the limited range in $d$. In contrast, the BCS and reduced models, with a single specific unpaired electron mode, still exhibit long range correlations. 


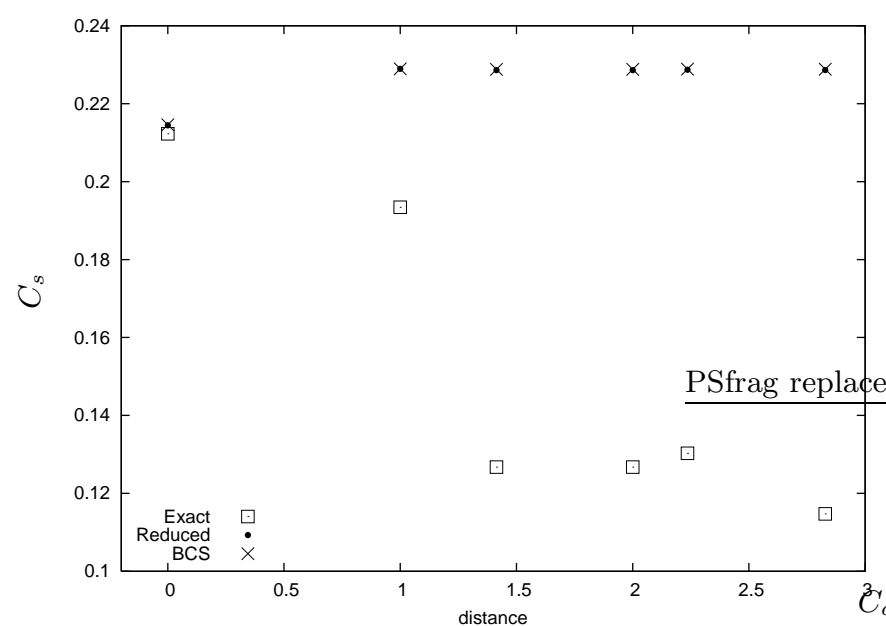

FIG. 12: The $s$-wave correlators as a function of distance (two dimensions, $N_{e}=16$ )

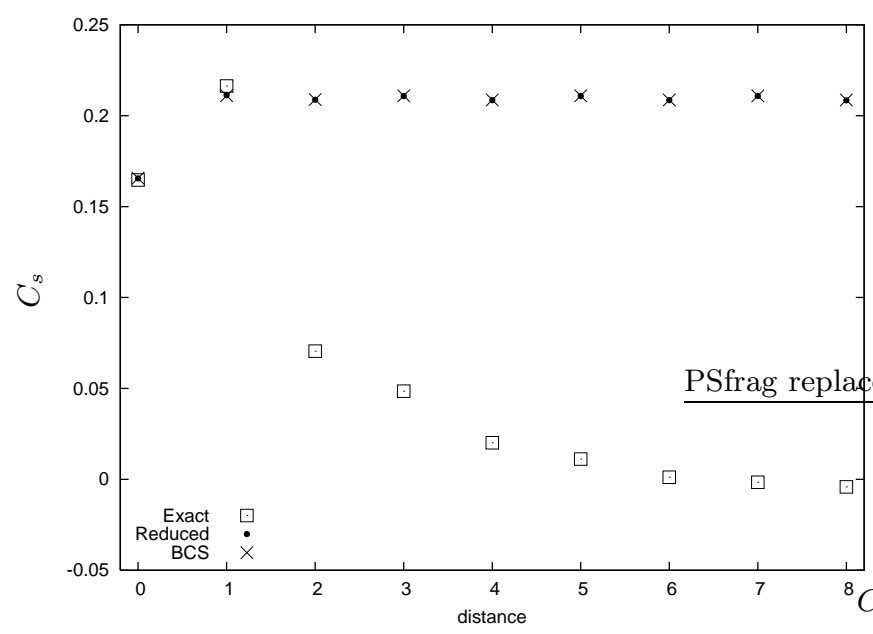

FIG. 13: The $s$-wave correlators as a function of distance (one dimension, $\left.N_{e}=15\right)$.

The $p$-wave correlators, $C_{p_{x}}(d)$, are plotted and compared in Figs. 15 16, 17] and 18 for different fillings and dimensions. Both interesting and somewhat surprising is that the differences between the BCS and reduced correlators are now discernable, especially in 2 dimensions. Since these two model wavefunctions are identical to within 1 part in $10^{3}$, this indicates that the $p$-wave correlator is a very sensitive wavefunction probe as this correlator's matrix elements are dominated by a very small portion of the state vector. This result also reflects the small $p$-wave correlator magnitude. None of the models exhibit strong long range order or significant $p_{x}$ correlations. Finally, the differences between Hubbard and reduced $p$-wave correlators are dramatic for both one and two dimensions with even or odd filling.

Finally, the $d$-wave correlators, $C_{d_{x^{2}-y^{2}}}$, were calculated in two dimensions (Figs. 19] and [20). As with the $p$-wave correlators, the magnitudes are small for non-zero $d$ and the differences between the Hubbard and reduced

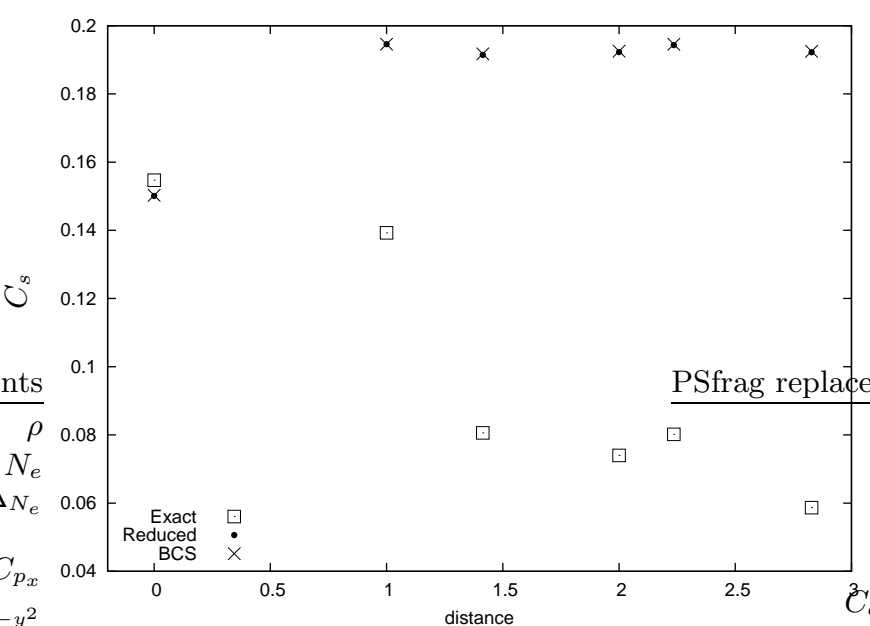

FIG. 14: The $s$-wave correlators as a function of distance (two dimensions, $N_{e}=15$ ).

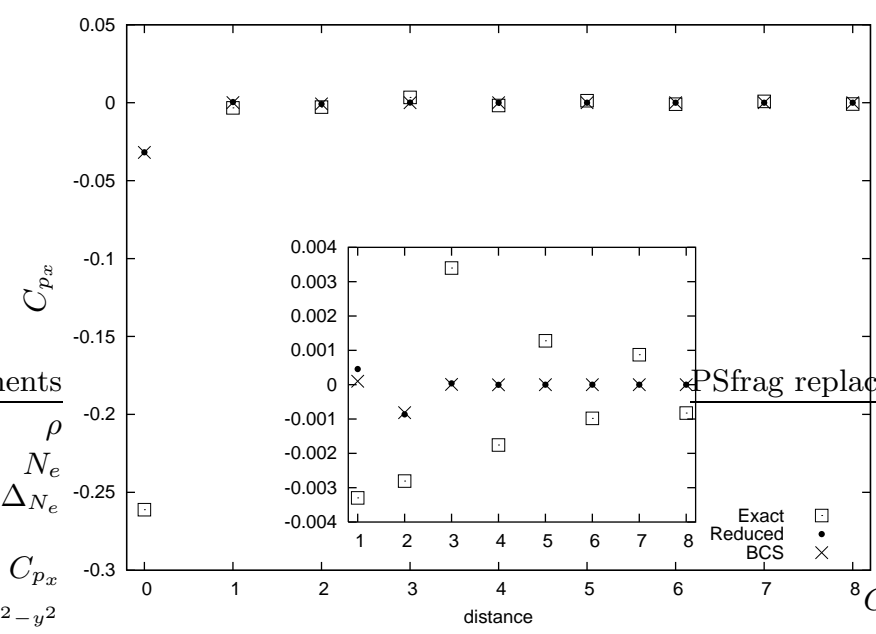

FIG. 15: The $p$-wave correlators as a function of distance (one dimension, $\left.N_{e}=16\right)$.

correlators are dramatic. The differences between the reduced and BCS correlators are again present. 


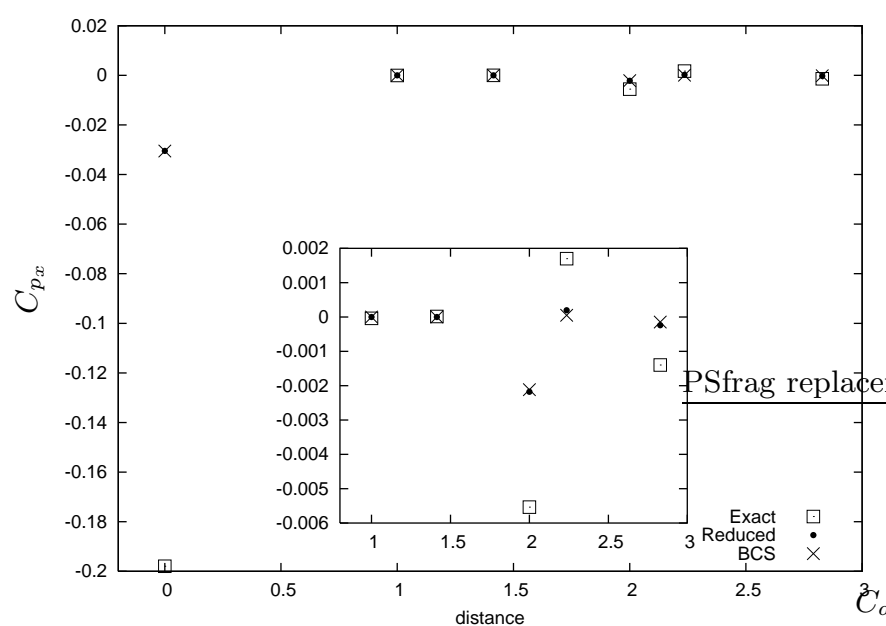

FIG. 16: The $p$-wave correlators as a function of distance (two dimensions, $N_{e}=16$ ).

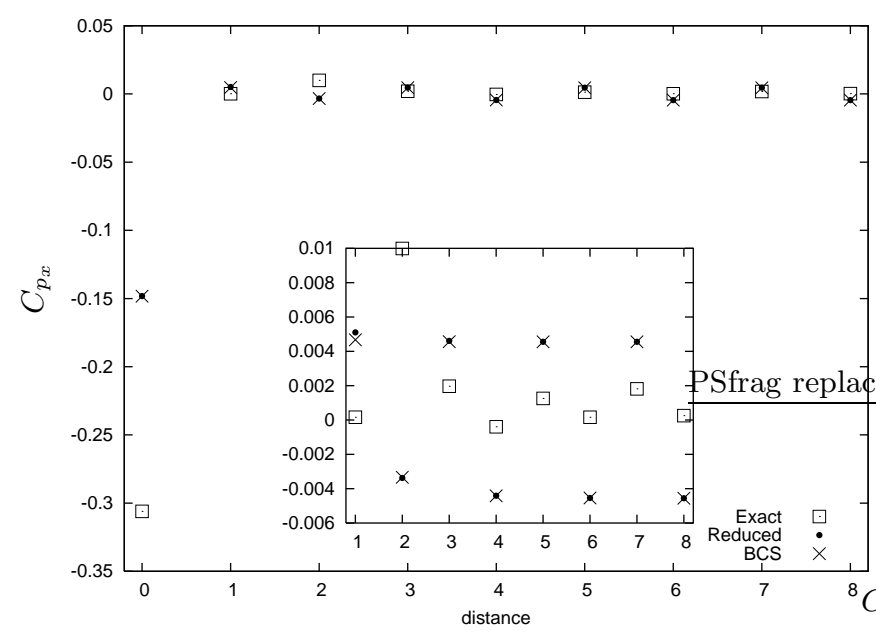

FIG. 17: The $p$-wave correlators as a function of distance (one dimensions, $N_{e}=15$ ).

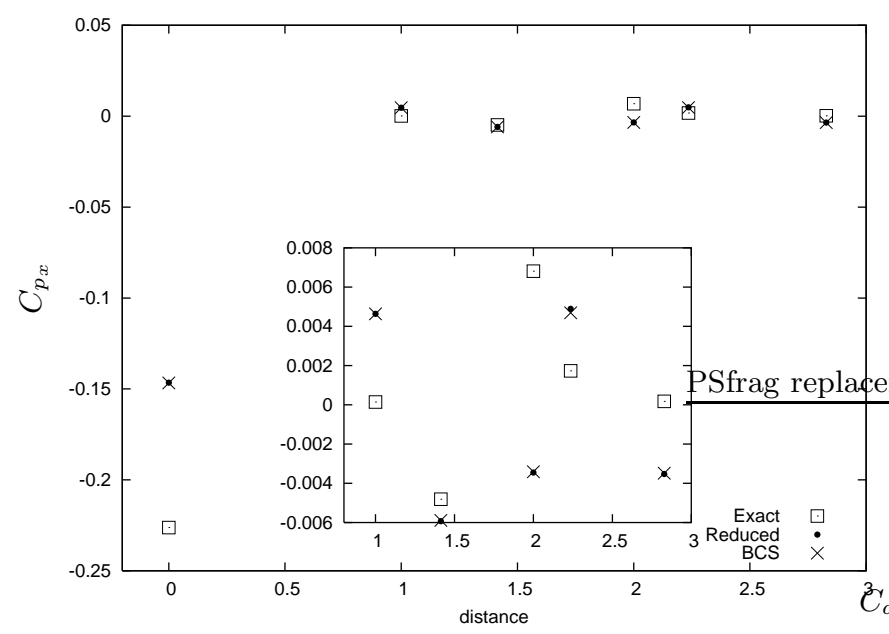

FIG. 18: The $p$-wave correlators as a function of distance (two dimensions, $N_{e}=15$ ).

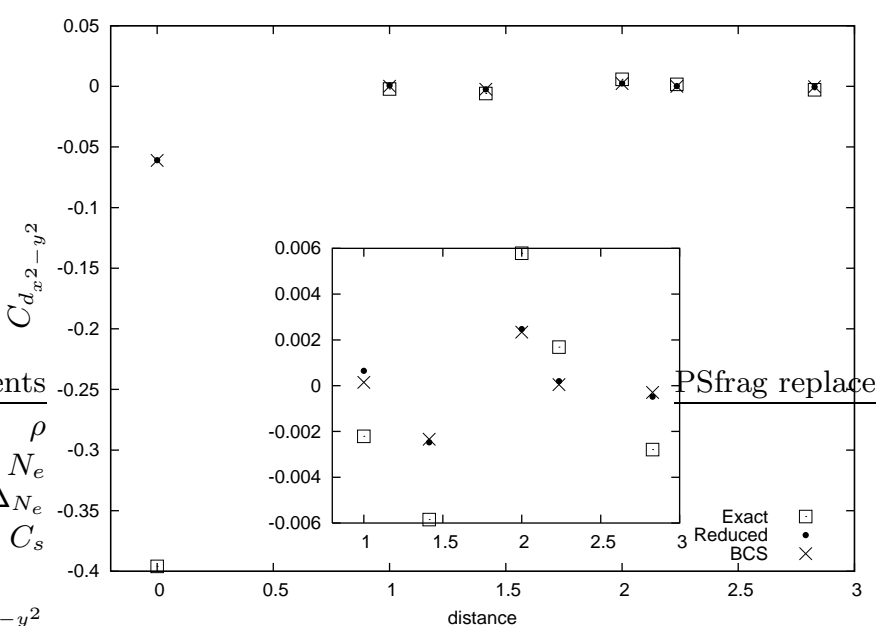

FIG. 19: The $d$-wave correlators as a function of distance (two dimensions, $N_{e}=16$ ).

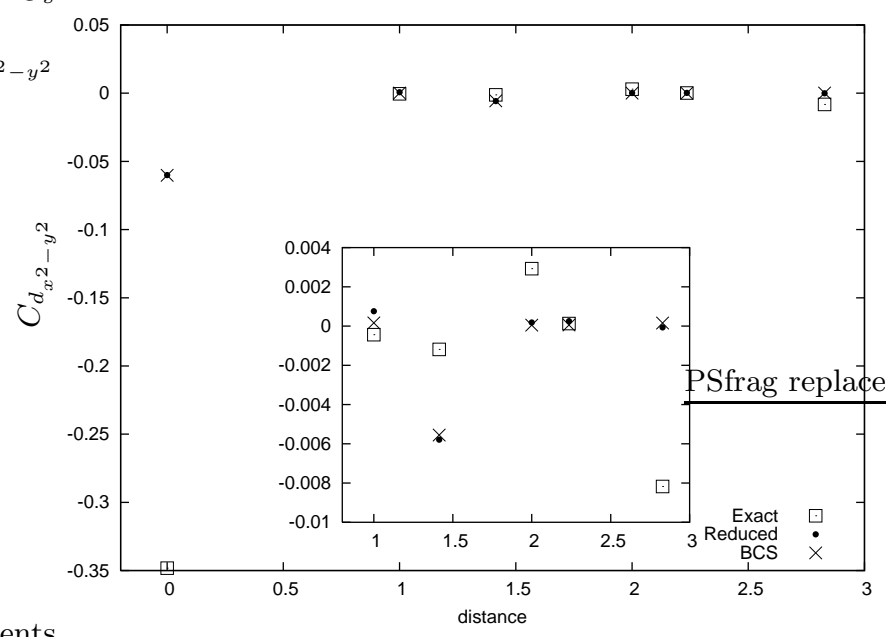

FIG. 20: The $d$-wave correlators as a function of distance (two dimensions, $N_{e}=15$ ). 


\section{CONCLUSIONS}

The major thrust of this work has been to further numerically assess the accuracy of the BCS approximation. By applying this approximation to multi-electron systems governed by both the Hubbard and a simpler, reduced Hamiltonian, each exactly diagonalizable, it has been documented that the BCS provides an excellent description of ground state energies in both one and two dimensions. However, the BCS single particle energy gaps are less accurate, in both one and two dimensions, especially near half filling. This undermines previous assertions that the BCS approximation improves with increasing dimension. Further, propagators, overlaps and correlators have been found to be quite sensitive to both interactions and approximations as the BCS and reduced model predictions are appreciably different than the Hubbard results. The slow decrease of the Hubbard and reduced Hamiltonian ODLRO propagators and correlator functions with increasing lattice separation is evidence for superconductivity. However correlations, and thus the degree of superconductivity, are much stronger in the reduced model. Finally, the BCS method extremely accurately reproduces all reduced model observables.

In summary, our results challenge the validity of the
BCS method as a good, comprehensive approximation to the attractive Hubbard model. While the Hubbard and BCS correlators both exhibit long-range order, their variations with distance are quite different. Because agreement between correlators and inner-product overlaps improves to some extent with dimension, perhaps in three dimensions the BCS method may yet be a very accurate alternative to exact Hubbard model wavefunctions but further study is necessary. Finally, for physical systems where the interactions embodied in the reduced Hamiltonian dominate, the BCS approximation will be a very accurate approximation scheme permitting significantly more ambitious numerical investigations.

\section{Acknowledgments}

The authors would like to thank Dean Lee, Frank Marsiglio, Lubos Mitas, Greg Recine and Thomas Schaefer for informative and fruitful communications. Computer time provided by Applied Electronics Lab, Stevens Institute of Technology is also appreciated. This work was supported in part by grants DOE DE-FG02-97ER41048 and NSF DMS-0209931.
1 J. Bardeen, L.N. Cooper and J.R. Schrieffer, Phys. Rev. 108, 1175 (1957).

2 N.N Bogoliubov, Nuovo Cimento 7, 794 (1958).

3 J.G. Valatin, Nuovo Cimento 7, 843 (1958).

${ }^{4}$ K. Dietrich, H.J. Mang and J.H. Pradal, Phys. Rev. 135, B22 (1964).

${ }^{5}$ F.J. Llanes-Estrada and S.R. Cotanch, Phys. Rev. Lett. 84, 1102 (2000).

6 F. Marsiglio, Phys. Rev. B55, 575 (1997).

7 F. Braun and J. von Delft, Phys. Rev. Lett. 81, 4712 (1998).

8 K. Tanaka and F. Marsiglio, Phys. Rev. B60, 3508 (1999).

9 W. Fettes, I. Morgenstern and T. Husslein, Int. J. Mod. Phys. C8, 1037 (1997).

10 E.H. Lieb and F.Y. Wu, Phys. Rev. Lett. 20, 1445 (1968).
11 J.R. Schrieffer, Theory of Superconductivity (W.A. Benjamin Inc., New York, NY 1964).

12 N.D. Mermin and H. Wagner, Phys. Rev. Lett. 17, 1133 (1966) and D. Ghosh, Phys. Rev. Lett. 27, 1584 (1971) proved that expectation value is zero for spin wave operators in the Heisenberg and Hubbard models, respectively. D. Jasnow and M.E. Fisher, Phys. Rev. B3, 907 (1971) proved, by the same methods, that a nonzero ODLRO is explicitly forbidden in the Heisenberg model.

13 The high $U$ expansion of the Bethe ansatz is given by M. Takahashi, Thermodynamics of one-dimensional solvable models (Cambridge University Press, Cambridge, UK 1999). By symmetry, $N_{e \uparrow}=N_{e \downarrow}$, with $U<0$, is equivalent to $N_{e \uparrow}+N_{e \downarrow}=V$, with $U>0$. 